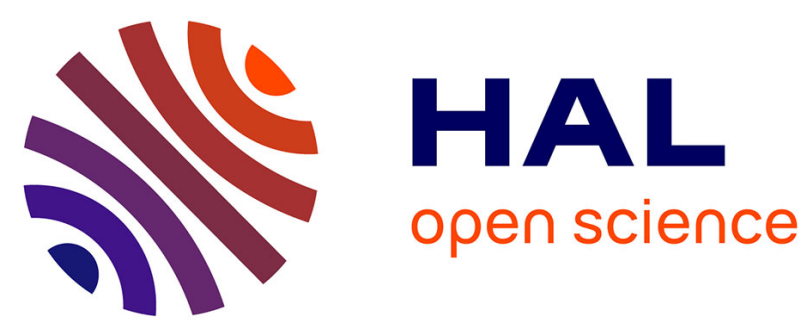

\title{
Expérience d'accélération d'électrons par onde de battement à l'INRSÉnergie
}

\author{
F. Martin, J.P. Matte, H. Pepin, N.E. Ebrahim
}

\section{To cite this version:}

F. Martin, J.P. Matte, H. Pepin, N.E. Ebrahim. Expérience d'accélération d'électrons par onde de battement à l'INRSÉnergie. Revue de Physique Appliquée, 1988, 23 (9), pp.1495-1504. 10.1051/rphysap:019880023090149500 . jpa-00245976

\section{HAL Id: jpa-00245976 https://hal.science/jpa-00245976}

Submitted on 1 Jan 1988

HAL is a multi-disciplinary open access archive for the deposit and dissemination of scientific research documents, whether they are published or not. The documents may come from teaching and research institutions in France or abroad, or from public or private research centers.
L'archive ouverte pluridisciplinaire HAL, est destinée au dépôt et à la diffusion de documents scientifiques de niveau recherche, publiés ou non, émanant des établissements d'enseignement et de recherche français ou étrangers, des laboratoires publics ou privés. 


\title{
Expérience d'accélération d'électrons par onde de battement à l'INRS- Énergie
}

\author{
F. Martin, J. P. Matte, H. Pepin et $\left({ }^{1}\right)$ N. E. Ebrahim \\ INRS-Energie, B.P. 1020, Varennes, Québec, Canada, J0L-2P0 \\ (1) AECL, Chalk River Nuclear Lab., Chalk River, Ontario, Canada
}

(Reçu le 15 octobre 1987, accepté le 19 mai 1988)

\begin{abstract}
Résumé. - Nous avons mis au point une nouvelle approche pour vérifier expérimentalement l'accélération d'électrons par onde de battement. Il y a plusieurs éléments originaux dans cette approche. Un laser $\mathrm{CO}_{2}$ de haute intensité, opérant simultanément à deux fréquences, est utilisé pour produire un plasma par ionisation laser d'un gaz basse pression avant de stimuler l'onde de battement lorsque la résonance $\left(\omega_{\mathrm{pe}}=\Delta \omega\right)$ est atteinte. Une source externe d'électrons de haute énergie $(0,2 \mathrm{MeV} \leqslant E \leqslant 1,0 \mathrm{MeV})$ provenant d'un plasma créé par irradiation d'une cible solide avec un laser $\mathrm{CO}_{2}$ est utilisée. Une gamme d'énergie restreinte $(\Delta E / E=0,25 \%)$ de ces électrons est focalisée par un spectrographe dipolaire pour être injectée dans le plasma, parallèlement à l'onde de battement. Cette source d'électrons de haute intensité $\left(=10^{8} \mathrm{el} . / \mathrm{cm}^{2}-\mathrm{keV}\right)$, pulsée $\left(\tau_{\mathrm{r}}=0,3 \mathrm{~ns}\right)$ peut facilement être synchronisée avec la production du plasma et est appropriée pour permettre une discrimination facile des électrons accélérés par l'onde de battement. Des mesures de bandes latérales anti-Stokes $(8,7 \mu \mathrm{m})$ servent à optimiser l'amplitude de l'onde plasma de résonance. Des mesures préliminaires indiquent une accélération effective de $1,0 \mathrm{GeV} / \mathrm{m}$ sur une longueur de plasma de $1,5 \mathrm{~mm}$.
\end{abstract}

\begin{abstract}
We have developed a means to experimentally verify electron acceleration by the beat-wave concept. There are many original elements in our approach. A high intensity $\mathrm{CO}_{2}$ laser, operating simultaneously at two frequencies, serves to produce a plasma by laser ionization of a low pressure gas before pumping the resonant plasma wave when resonance $\left(\omega_{\mathrm{pe}}=\Delta \omega\right)$ is reached. A separate source of high energy electrons $(0.2 \mathrm{Mev} \leqslant E \leqslant 1.0 \mathrm{MeV})$ is extracted from a $\mathrm{CO}_{2}$ laser produced plasma on a solid target. A narrow energy band $(\Delta E / E=0.25 \%))$ of these electrons is focussed by a magnetic dipole spectrograph and injected into the resonant plasma, parallel to the beat wave. This high intensity $\left(=10^{8} \mathrm{el} . / \mathrm{cm}^{2}-\mathrm{keV}\right)$, short pulse $(\tau=0.3 \mathrm{~ns})$, source of electrons can easily be synchronized to the production of the resonant plasma and allows a good discrimination of the electrons accelerated by the beat wave. Anti-Stokes sideband measurements $(8.7 \mu \mathrm{m})$ allow the amplitude of the resonant plasma wave to be optimized. Preliminary measurements indicate an acceleration efficiency of $1 \mathrm{GeV} / \mathrm{m}$ over a plasma length of $1.5 \mathrm{~mm}$.
\end{abstract}

\section{Introduction.}

Les concepts d'accélérateurs d'électrons de haute énergie basés sur l'utilisation d'un laser de haute intensité peuvent, en principe, augmenter le gradient d'accélération de la limite actuelle de $10 \mathrm{MeV} / \mathrm{m}$ pour les accélérateurs « conventionnels» jusqu'au niveau de $1 \mathrm{GeV} / \mathrm{m}$ [1] ou plus. Parmi les nombreux concepts qui ont été proposés, ceux qui sont basés sur l'utilisation d'un plasma comme milieu accélérateur offrent l'avantage de pouvoir supporter un champ électrique très élevé. L'un de ces concepts est l'accélérateur par onde de battement [2] où l'on utilise une impulsion laser à deux longueurs d'ondes $\left(\omega_{1}, \omega_{2}\right)$ pour exciter une onde plasma résonante $\left(\omega_{\mathrm{pe}}=\Delta \omega=\omega_{1}-\omega_{2}\right)$. Cette onde a une vitesse de phase voisine de la vitesse de la lumière $v_{\phi}=$ $c\left(1-\left(\omega_{\mathrm{pe}} / \omega\right)^{2}\right)^{1 / 2}$ et peut atteindre une amplitude élevée $(\Delta n / n \leqslant 1)$ de sorte que le champ électrique longitudinal associé à cette onde peut accélérer des électrons jusqu'à de très hautes énergies.

Cet article décrit notre approche pour vérifier expérimentalement l'accélération d'électrons par onde de battement. La première section décrit le laser $\mathrm{CO}_{2}$ utilisé pour ces expériences. La seconde section décrit l'ionisation par laser $\mathrm{CO}_{2}$ qui sert à produire le plasma dans lequel l'onde de battement sera stimulée. Cette section couvre les mécanismes 
d'ionisation par laser et les méthodes de mesure de la densité électronique. La troisième section traite de la production de l'onde de battement par le laser $\mathrm{CO}_{2}$ à deux fréquences. Cette section décrit les mécanismes de saturation de l'onde de battement et donne une estimation de la largeur de bande de la résonance. On y discute aussi les mesures de bandes latérales qui permettent, à l'aide de ces modèles, d'estimer l'amplitude de saturation. La quatrième section décrit la source d'électrons qui sert à produire un «faisceau » d'électrons monoénergétiques pulsé pour injection dans l'onde de battement. La cinquième section décrit l'expérience d'accélération d'électrons ainsi que les résultats préliminaires obtenus. L'article se termine par un sommaire.

\section{Laser $\mathrm{CO}_{2}$.}

Les expériences d'ionisation et de pompage de l'onde plasma à la résonance ont été effectuées avec un laser $\mathrm{CO}_{2}$. Ce laser est composé de deux oscillateurs à modes-bloqués qui peuvent être synchronisés indépendamment sur n'importe laquelle des raies du spectre de gain du $\mathrm{CO}_{2}$. La synchronisation fine (sur l'échelle de la nanosec) entre les deux trains d'impulsions se fait en ajustant la phase du signal RF allant à un des modulateurs. Le train d'impulsions sortant de chacun de ces oscillateurs est combiné en utilisant un prisme de $\mathrm{NaCl}$. Une impulsion à deux fréquences est découpée en utilisant un commutateur électrooptique en AsGa. Cette impulsion, ayant une durée d'une nanoseconde, est amplifiée ( 2 passages) dans un amplificateur à pression atmosphérique, passe par un filtre spatial avant l'amplification finale ( 2 passages) dans un amplificateur à trois (3) atmosphères.

Cette chaîne laser, fonctionnant à une seule fréquence $(10 \mathrm{P} 20, \lambda=10,6 \mu \mathrm{m})$ produit une impulsion de 75 joules ayant une durée d'une nanoseconde (largeur à mi-hauteur). Opérant à deux longueurs d'ondes, soit les raies 9P20 $(\lambda=9,54 \mu \mathrm{m})$ et 10P16 $(\lambda=10,54 \mu \mathrm{m})$, ce système produit des impulsions lasers de 30 joules à chaque longueur d'onde. Le temps de montée de l'impulsion laser est estimé à $0,3 \mathrm{~ns}$. Le faisceau laser de $10 \mathrm{~cm}$ de section est focalisé par un miroir parabolique hors-axe $f / 6$ de $60 \mathrm{~cm}$ de focale, produisant une tache de $150 \mu \mathrm{m}$ de diamètre à mi-énergie ce qui correspond à une intensité laser moyenne de 4,0 $\times 10^{14}$ Watts $/ \mathrm{cm}^{2}$.

\section{Source plasma.}

Pour une amplitude $(\Delta n / n)$ donnée de l'onde de battement, le champ électrique longitudinal est proportionnel à la fréquence plasma $\omega_{\mathrm{pe}} \sim n_{\mathrm{e}}^{1 / 2}$. Les lasers à haute intensité actuellement disponibles opérant à deux longueurs d'onde, ne peuvent fonctionner avec un écart de fréquence supérieur à $3,0 \times 10^{12}$ Hertz ce qui correspond à une densité électronique résonnante de $10^{17} \mathrm{~cm}^{-3}$. Les décharges à striction $Z$ et $\theta$ peuvent atteindre ces densités électroniques et ont été utilisées comme source plasma pour générer l'onde de battement [3]. Notons cependant que ces sources de plasma ont besoin de synchronisation externe avec le laser, qu'ils impliquent des champs magnétiques qui peuvent dévier ou défocaliser un faisceau d'électrons et que le plasma est beaucoup plus grand que la dimension de l'onde de battement.

L'ionisation par laser d'un gaz basse pression peut aussi produire un plasma à la densité électronique résonnante. L'étendue du plasma se limite à la région de haute intensité du volume focal, c'est-àdire limité à la région où est stimulée l'onde de battement. Il n'y a pas de synchronisation nécessaire puisque c'est le même laser qui ionise le gaz basse pression pour ensuite stimuler l'onde de battement lorsque la densité résonnante est atteinte. La pression de remplissage du gaz permet d'ajuster avec précision la densité électronique et l'intensité laser détermine le délai entre la production du plasma résonnant et le maximum de l'impulsion laser.

Il y a deux mécanismes d'ionisation [4] par laser d'un gaz basse pression : l'ionisation par effet tunnel (ou multiphotonique aux longueurs d'onde plus courtes) et l'ionisation collisionnelle. Le taux d'ionisation collisionnelle des électrons oscillant dans le champ électrique du laser n'est pas suffisant à basse pression $\left(P=1,4\right.$ Torr $\left.\mathrm{H}_{2}\right)$ pour produire un plasma de haute densité $\left(n_{\mathrm{e}}=1 \times 10^{17} \mathrm{~cm}^{-3}\right)$ sur une échelle de temps inférieure à la nanoseconde. L'ionisation par effet tunnel peut produire un plasma de cette densité à un taux très rapide pourvu que l'intensité laser soit supérieure à $I \geqslant 10^{14}$ Watts $/ \mathrm{cm}^{2}$.

2.1 MÉCANISMES D'IONISATION ET MODÉLISATION. - Un modèle simple [15] permet de mieux comprendre les procédés d'ionisation et d'en déterminer l'évolution temporelle. Ce modèle incorpore le taux d'ionisation par effet tunnel de Keldysh [4] et le taux d'ionisation de Lotz [4] qui modélise l'ionisation par impact dû aux électrons qui ont été accélérés par le champ électrique du laser. Des calculs ont aussi été effectués avec un taux de Keldysh modifié. Des mesures récentes de Yergeau [5] sur l'ionisation par laser $\mathrm{CO}_{2}$ intense de gaz à très basse pression indiquent qu'une augmentation d'un facteur $10^{8} \mathrm{du}$ taux d'ionisation de Keldysh est nécessaire pour expliquer ses résultats expérimentaux.

L'évolution temporelle de la densité électronique d'après ce modèle est représentée figure 1 . Une dépendence temporelle de l'intensité laser avec un temps de montée de 0,3 ns et une largeur à mihauteur de 1,0 ns a été utilisée. L'intensité maximum est de $I=2 \times 10^{14} \mathrm{Watts} / \mathrm{cm}^{2}$. Les pressions pour les deux gaz utilisés dans ces calculs ont été choisies pour obtenir une densité électronique de $n_{\mathrm{e}}=$ $1 \times 10^{17} \mathrm{~cm}^{-3}$ durant l'impulsion laser. 


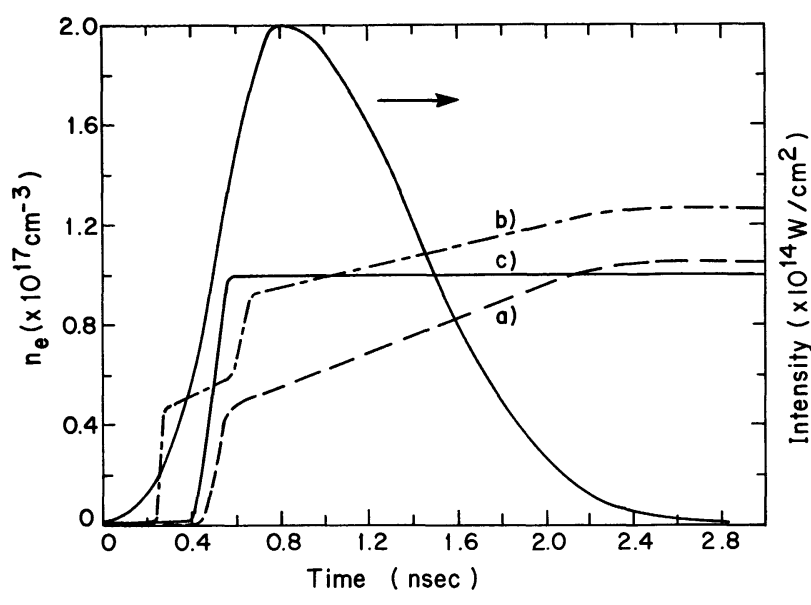

Fig. 1. - L'évolution temporelle de la densité électronique d'après le modèle d'ionisation. La dépendence temporelle de l'intensité de l'impulsion laser y est représentée. La courbe a) correspond à 0,65 torr d'azote $\left(\mathrm{N}_{2}\right)$, la courbe $b$ ) correspond à 0,65 torr d'azote $\left(\mathrm{N}_{2}\right)$ et un taux de Keldysh augmenté et la courbe c) correspond à 1,4 torr d'hydrogène $\left(\mathrm{H}_{2}\right)$.

[Temporal evolution of the electron density from an ionization model. The temporal dependence of the laser intensity is also shown. Curve a) corresponds to a nitrogen $\left(\mathrm{N}_{2}\right)$ pressure of 0,65 torr, curve $\mathrm{b}$ ) corresponds to a nitrogen $\left(\mathrm{N}_{2}\right)$ pressure of 0,65 torr and an augmented Keldysh ionization rate and curve c) corresponds to a hydrogen $\left(\mathrm{H}_{2}\right)$ pressure of 1,4 torr.]

D'après ce modèle, l'ionisation du gaz basse pression ne débute que lorsque l'intensité laser a dépassé le seuil pour l'ionisation par effet tunnel. Alors la densité électronique augmente à un taux très élevé car l'ionisation par effet tunnel arrache le premier électron en moins de $0,15 \mathrm{~ns}$. Le seuil en intensité est plus bas pour l'hydrogène que pour l'azote (comparer les courbes a et c) puisque le potentiel d'ionisation du premier électron est plus bas. Il va sans dire que d'arracher le premier électron à l'hydrogène correspond à un plasma complètement ionisé ce qui explique pourquoi la densité électronique reste constante jusqu'à la fin de l'impulsion laser. Le fait d'augmenter le taux d'ionisation de Keldysh pour le cas de l'hydrogène ne ferait que diminuer le seuil d'ionisation sans pour autant changer la valeur de saturation de la densité électronique. Par contre, l'augmentation du taux d'ionisation de Keldysh représente une importante contribution dans le cas de l'azote $\left(\mathrm{N}_{2}\right)$ (courbe b) car non seulement le seuil d'ionisation est abaissé mais l'ionisation par effet tunnel contribue à arracher le second électron. Par la suite, l'ionisation collisionnelle domine mais avec un taux d'ionisation beaucoup plus lent, de l'ordre de $0,3 \times 10^{17} / \mathrm{cm}^{3}$-ns, n'atteignant jamais l'ionisation complète.

Ce modèle simple démontre que l'ionisation par laser peut produire une densité électronique de $n_{\mathrm{e}}=1 \times 10^{17} \mathrm{~cm}^{-3}$ durant le temps de montée de 0,3 ns d'une impulsion laser. Par contre, pour être consistant avec les mesures d'interférométrie et de résonance, discutées dans la prochaine section, le modèle d'ionisation doit utiliser le taux d'ionisation de Keldysh augmenté ainsi qu'un taux d'ionisation de Lotz plus élevé. Ceci ne démontre pas que le taux augmenté est exact mais indique plutôt que la théorie d'ionisation par effet tunnel de Keldysh ne permet pas d'expliquer nos résultats expérimentaux. Il y a aussi plusieurs procédés d'ionisation collisionnelle qui ne sont pas pris en compte dans le taux d'ionisation de Lotz. L'usage d'un facteur numérique ajouté au taux d'ionisation n'est qu'une tentative pour expliquer nos résultats expérimentaux dans un domaine où les processus d'ionisation ne sont pas bien connus ou compris. Ce modèle se limite à une description phénoménologique (qualitative) de l'évolution temporelle de la densité électronique lors de l'ionisation par laser de gaz basse pression.

2.2 Mesure DE DENSITÉ ÉleCtroniQue. - La mesure de la densité électronique de plasmas générés par ionisation laser de gaz basse pression pose certains problèmes particuliers. Ces plasmas ont une densité électronique élevée, mais leur durée de vie est courte (nanoseconde) et leurs dimensions se limitent au volume focal (diamètre $=0,2 \mathrm{~mm}$, longueur $=1,5 \mathrm{~mm}$ ). La diffusion Thomson permet de mesurer la valeur absolue du plateau de densité électronique [6] avec une grande précision mais ne peut donner la distribution spatiale que de manière très grossière. L'interférométrie, utilisant une impulsion $\mathrm{CO}_{2}$ sonde, donne un résolution acceptable de la valeur absolue de la densité électronique et de sa distribution spatiale. La résolution de cette méthode de mesure est limitée par le faible déphasage (moins d'une frange) de la sonde $\mathrm{CO}_{2}$ dû au faible produit $\left(n_{\mathrm{e}} \times\right.$ longueur du plasma). Les mesures de Bremsstrahlung émis par le plasma peuvent donner la distribution relative de densité électronique [7]. Ce diagnostic ne sera pas décrit dans cet article.

Les mesures d'interférométrie [15] ont été effectuées avec un laser $\mathrm{CO}_{2}$ haute intensité fonctionnant à une seule longueur d'onde. Une partie du faisceau intense $(4,0 \%)$ sert de faisceau sonde. Le déphasage dû au plasma est mesuré par un interféromètre Mach-Zender. L'image du plasma est obtenue par un télescope afocal ayant un grossissement de deux. L'interférogramme est enregistré en brûlant une photo Polaroid (MD). La durée d'impulsion du faisceau sonde (1 ns) ne permet pas une résolution temporelle durant la phase d'ionisation. Par conséquent, les interférogrammes ont été enregistrés à $2,0 \mathrm{~ns}$ et 5,0 ns après l'impulsion $\mathrm{CO}_{2}$ intense qui produit le plasma. La recombinaison (électron-ion) et la diffusion du plasma sont négligeables sur cette échelle de temps de sorte que l'interférométrie 
mesure la valeur de saturation de la distribution de la densité électronique. Les interférogrammes sont déconvolués par inversion d'Abel pour obtenir la distribution axiale et radiale de densité électronique. La gamme de pression étudiée est de 1,0 torr $\leqslant$ $P \leqslant 2,0$ torr pour l'hydrogène $\left(\mathrm{H}_{2}\right)$ et 0,3 torr $\leqslant P \leqslant 2,0$ torr pour l'air (surtout $\mathrm{N}_{2}$ ). L'intensité laser est dans la gamme 2,0-4,0 $\times 10^{14}$ Watts $/ \mathrm{cm}^{2}$.

Ces mesures indiquent que la dimension du plasma est à toute fin pratique indépendante de la pression de remplissage pour ces deux gaz dans la gamme de pression considérée ici. Le plasma est cylindrique avec un diamètre de $0,2 \mathrm{~mm}$ et une longueur de $2,0 \mathrm{~mm}$ à mi-hauteur. Ces dimensions sont légèrement plus grandes que le volume focal, soit $0,15 \mathrm{~mm}$ de diamètre et de $1,5 \mathrm{~mm}$ de profondeur focale. La précision de la mesure ne permet pas de résoudre les variations de densité à l'intérieur du volume focal. La valeur moyenne de la densité électronique à l'intérieur du volume focal constitue alors une mesure de la densité crête. L'excursion de la densité électronique à l'intérieur du volume focal donne la précision de la mesure et sert de barre d'erreur.

Dans le cas de l'hydrogène, la densité électronique crête correspond à l'ionisation complète du gaz de sorte qu'il existe une dépendence linéaire entre la densité électronique crête et la pression de remplissage. Pour ce qui est de l'air, la dépendence de la densité électronique crête en fonction de la pression de remplissage pour un délai sonde de $2,0 \mathrm{~ns}$ est représentée sur la figure 2 . La courbe solide corres-

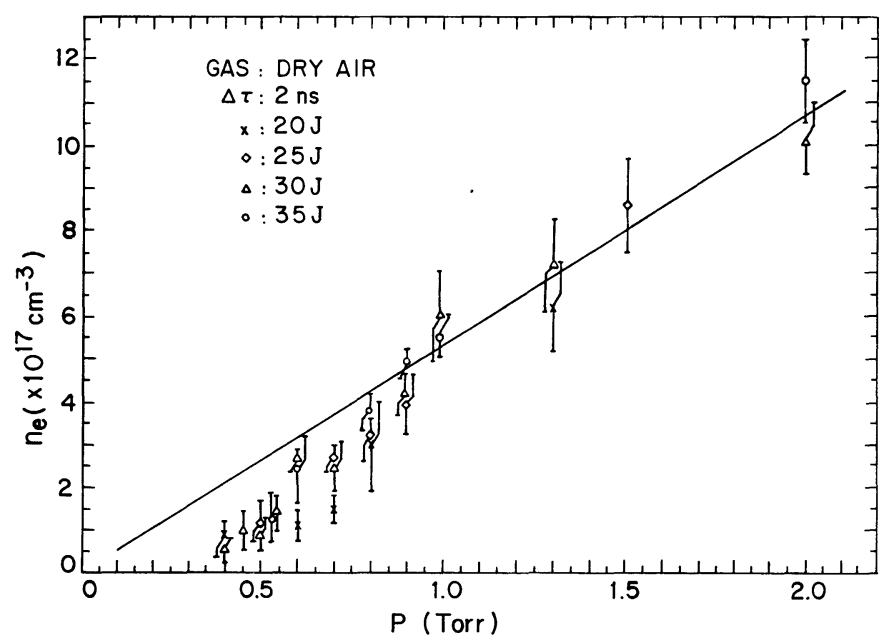

Fig. 2. - Dépendence de la densité électronique crête mesurée par interférométrie en fonction de la pression de remplissage pour l'air. Le délai du faisceau sonde est de $2,0 \mathrm{~ns}$. La courbe solide correspond à l'ionisation complète.

[Dependence of the peak electron density as a function of the filling pressure for air. The probe delay is $2,0 \mathrm{~ns}$. The solid curve corresponds to complete ionization.] pond à l'ionisation complète. Les mesures prises à un délai de 5,0 ns donnent la même dépendence de la densité crête en fonction de la pression de remplissage indiquant que la densité électronique ne change pas appréciablement sur l'échelle de temps de la nanoseconde confirmant l'hypothèse faite cidessus. On observe que l'ionisation est partielle à basse pression, le facteur d'ionisation augmentant avec la pression pour atteindre l'ionisation complète pour une pression de 1,0 torr d'air $\left(\mathrm{N}_{2}\right)$. La densité crête n'est pas sensible à l'énergie laser dans la gamme d'énergie rapportée. Une densité électronique de $n_{\mathrm{e}}=1,0 \times 10^{17} \mathrm{~cm}^{-3}$ est obtenue à une pression de 0,5 torr d'air correspondant à un facteur d'ionisation de $Z=3$. Ces mesures, ainsi que celles faites dans d'autres laboratoires [6], démontrent qu'un plasma produit par ionisation laser a les caractéristiques voulues (densité et dimensions) pour servir à la production d'une onde de battement.

Il va sans dire que le critère ultime pour ceci est de mesurer l'onde de battement. Ces mesures font l'objet de la prochaine section. Le modèle d'ionisation et les mesures de densité électronique permettent de dire que le plasma produit par ionisation laser $\mathrm{CO}_{2}$ intense est créé sur une échelle de temps très rapide (plus courte que l'impulsion laser), qu'une densité électronique de $n_{\mathrm{e}}=1,0 \times 10^{17} \mathrm{~cm}^{-3}$ peut être atteinte et que la dimension du plasma est légèrement supérieure au volume focale où l'onde de battement est généré. Les mesures de diffusion Thomson [6] montrent que la densité électronique d'un plasma créé par ionisation laser est très uniforme $(\delta n / n \leqslant 1 \%)$.

\section{Production de l'onde de battement.}

Lorsqu'une impulsion laser à deux longueurs d'onde est focalisée sur un plasma à la densité résonnante $\left(\Delta \omega=\omega_{\mathrm{pe}}\right)$, le couplage entre la force pondéromotrice du laser à la fréquence de battement et l'onde plasma est très efficace. Il y a plusieurs mécanismes de saturation $[10,11]$ qui vont limiter l'amplitude maximum de l'onde plasma sous la barrière théorique de $\Delta n / n \leqslant 1$. La question de l'amplitude de saturation ainsi que de la largeur de résonance sera traitée dans la première partie de cette section. La seconde partie couvrira les méthodes de mesures de l'onde de battement et la comparaison avec les modèles théoriques.

3.1 AMPLITUdE DE SATURATION ET LARGEUR DE RÉSONANCE. - Le mécanisme de saturation donnant l'amplitude maximum a été identifié par Rosenbluth et Liu [8] comme étant la désynchronisation relativiste due à l'augmentation de masse de l'électron oscillant dans le champ électrique de l'onde plasma. Cette théorie suppose la résonance exacte $\left(\Delta \omega=\omega_{\mathrm{pe}}\right)$. Tang, Sprangle et Sudan [8] ont élargi 
le modèle pour inclure les effets de désynchronisation $\left(\Delta \omega \neq \omega_{\mathrm{pe}}\right)$ démontrant que pour un faible écart de résonance, l'amplitude de saturation augmente de $50 \%$.

Mais il y a aussi d'autres mécanismes de saturation qui peuvent limiter l'onde à une amplitude inférieure à la limite de saturation relativiste. Lorsque l'intensité laser est faible, l'amortissement collisionnel peut limiter l'amplitude de saturation de l'onde de battement. Bingham et al. [9] ont fait des calculs utilisant une fréquence de collision qui tient compte non seulement du mouvement thermique des électrons mais aussi du mouvement oscillant des électrons dans le champ électrique du laser. Cet effet est quelque peu réduit (J. P. Matte et al. [9]) si l'on considère aussi le mouvement oscillant des électrons dans le champ électrique de l'onde de battement. Ces deux limites de saturation sont représentées figure 3 .

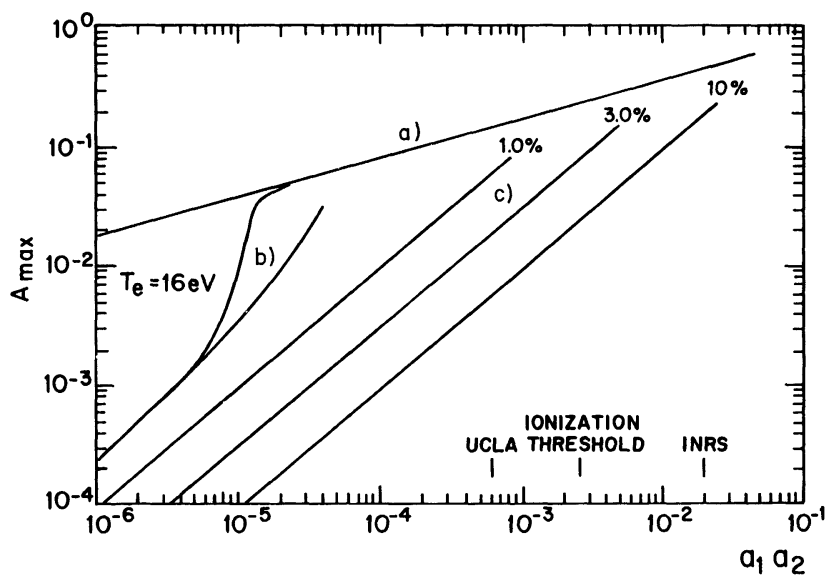

Fig. 3. - Amplitude de saturation suivant trois modèles : a) saturation relativiste de Rosenbluth et Liu, b) saturation incluant les effets collisionnels (la courbe du bas néglige l'oscillation des électrons dans l'onde plasma) et c) saturation due à la désynchronisation pour différentes valeurs d'écart de densité.

[Saturation amplitude from three models : a) relativistic saturation of Rosenbluth and Liu, b) saturation including collisional effects and c) saturation due to detuning as a function of density mismatch.]

Un autre facteur qui peut limiter l'amplitude de saturation est le désaccord entre la fréquence de battement du laser $(\Delta \omega)$ et la fréquence plasma $\left(\omega_{\mathrm{pe}}\right)$. Ce désaccord affecte aussi la phase de l'onde plasma par rapport au battement des deux fréquences laser, considération importante pour une application aux accélérateurs d'électrons. Deux facteurs peuvent contribuer à cette désynchronisation : un manque de contrôle sur la densité moyenne du plasma produisant une désynchronisation complète de l'onde plasma et une non-uniformité du plasma produisant une désynchronisation locale. Cette nonuniformité peut être une modulation spatiale de la densité électronique due au procédé d'ionisation ou à des ondes ioniques générées par le laser. La désynchronisation due à un écart de la densité moyenne du plasma a été traitée par Tang et al. [8]. La désynchronisation due à des inhomogénéités du plasma de période plus courte que l'onde de plasma a été traitée par Darrow et al. [10]. Il faut noter que ce second mode de désynchronisation, causée par le couplage Brillouin stimulé, est toujours présent dans un plasma où l'on focalise un laser.

L'inefficacité de pompage dû à une désynchronisation de la densité moyenne cause nécessairement une diminution de l'amplitude de saturation de l'onde plasma. La présence de modulation ionique dans le plasma cause aussi une diminution de l'amplitude de saturation de l'onde plasma : cette diminution est due au couplage entre l'onde plasma et la modulation ionique qui transfert de l'énergie de l'onde plasma à des modes auxilliaires à grand nombre d'onde $\left(k_{\mathrm{p}}=2 k_{\text {laser }}\right)$. L'amplitude de l'onde plasma est limitée parce que la perte d'énergie allant aux modes auxilliaires est plus grande que le transfert d'énergie venant du laser.

Il faut noter que ces deux procédés ont le même comportement phénoménologique, la désynchronisation sur toute la longueur de l'onde plasma de résonance n'étant que l'extension de la désynchronisation « locale » due à de très grandes périodes. Il s'avère donc qu'une description simple peut couvrir ces deux causes de saturation. Cette description est conforme à l'analyse plus rigoureuse qui a été faite par les auteurs mentionnés ci-dessus.

L'analyse simple d'un oscillateur forcé [11] peut être utilisée pour expliquer le principe de la décroissance de l'amplitude de saturation de l'onde plasma causée par la désynchronisation. Cette analyse est valide aux grandes valeurs de désynchronisation, c'est-à-dire pour des valeurs de désynchronisation telles que l'amplitude de saturation ne dépasse pas $20 \%$ de la saturation relativiste à cette intensité. Aux faibles valeurs de désynchronisation, il faut passer au modèle relativiste. Pour ce modèle simple, l'amplitude de saturation est donnée par l'expression :

$$
A_{\mathrm{sat}}=\frac{0,5 a_{1} a_{2}}{\left(4 \delta^{2}+\nu^{* 2}\right)^{1 / 2}}
$$

où $a_{\mathrm{i}}$ est la vitesse d'oscillation d'un électron dans le champ électrique du laser normalisé sur la vitesse lumière $\left(a_{\mathrm{i}}=9,0 \times 10^{-9}\left(I_{\mathrm{i}}\right)^{1 / 2}\right), I_{\mathrm{i}}\left[\right.$ Watts $\left./ \mathrm{cm}^{2}\right]$ est l'intensité laser $\mathrm{CO}_{2}, \quad \delta=\left(\omega_{\mathrm{pe}}-\Delta \omega\right) / \Delta \omega$ est le facteur de désynchronisation et $\nu^{*}=\nu / \Delta \omega$ est une fréquence d'amortissement normalisée. Cette expression s'emploie directement dans le cas d'une désynchronisation complète du plasma. Pour ce qui est du cas d'une onde ionique à période plus courte 
que l'onde plasma, la dépendence paramétrique est la même [10], mais le facteur de proportionnalité est maintenant $50 \%$ plus élevé $(0,75$ au lieu de 0,5$)$. Pour ce cas, $\delta$ devient proportionnel au carré de la modulation en densité de l'onde ionique. L'augmentation de l'amplitude de saturation par rapport à la désynchronisation complète se comprend par le fait que l'onde plasma est en résonance localement pour un certain nombre de points sur une période ce qui intuitivement permet un transfert plus efficace d'énergie.

L'amplitude de saturation correspondant à ces trois mécanismes est représentée à la figure 3 en fonction de la moyenne géométrique de l'intensité laser aux deux fréquences. Il est évident que pour la saturation collisionnelle et la saturation par désynchronisation pour une valeur $\delta$ fixe, lorsque l'intensité laser est suffisante, l'amplitude de saturation passe à la saturation relativiste. C'est-à-dire que le modèle n'est plus valide pour ce régime de paramètre.

La dépendence exacte de l'amplitude de saturation en fonction de la désynchronisation pour un plasma de densité uniforme a été développée par Tang, Sprangle et Sudan [8]. La figure 4 représente la courbe de résonance en fonction de la densité électronique calculée suivant ce modèle pour plusieurs valeurs du produit des vitesses d'oscillation. A la résonance exacte $n_{\mathrm{e}}=1,0 \times 10^{17} \mathrm{~cm}^{-3}$, ces calculs donnent le niveau de saturation relativiste de Rosenbluth et Liu [8]. Pour les amplitudes de saturation inférieure à $20 \%$ de la saturation relativiste, la dépendence en fonction de la désynchronisation est

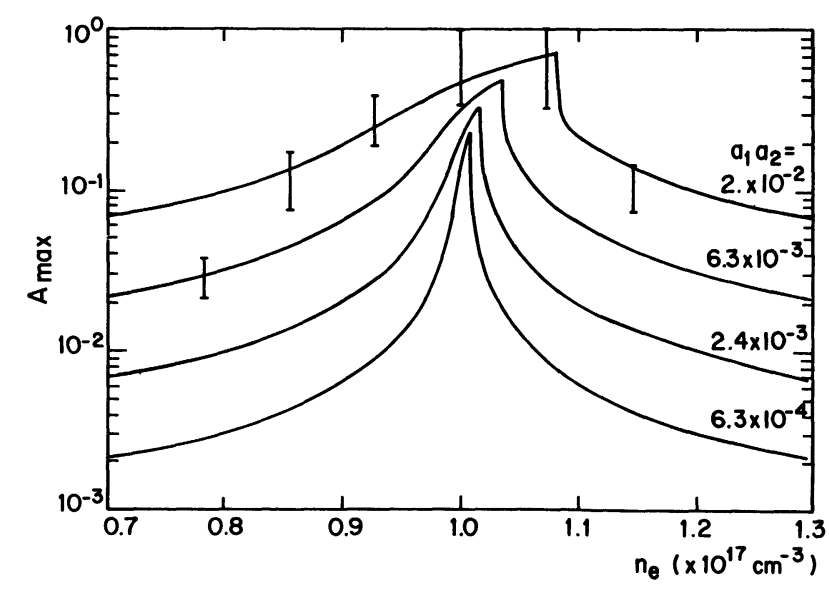

Fig. 4. - Amplitude de saturation de l'onde plasma en fonction de la densité électronique d'après le modèle de Tang et al. La densité électronique de résonance est de $1,0 \times 10^{17} \mathrm{~cm}^{-3} \cdot a_{\mathrm{i}}$ est la vitesse d'oscillation des électrons dans l'onde laser normalisée à la vitesse lumière.

[Saturation amplitude of the plasma wave as a function of the electron density. The resonant electron density is $1,0 \times 10^{17} \mathrm{~cm}^{-3}$.] identique à celle du modèle simple de Landau et Lifchitz. Il est à remarquer aussi que pour une faible désynchronisation positive $\left(\omega_{\mathrm{pe}} \geqslant \Delta \omega\right)$, l'amplitude de saturation est plus grande de $50 \%$ par rapport au niveau de saturation correspondant à la résonance exacte. Toute augmentation de la densité électronique au-delà de ce niveau optimal entraîne une chute importante du niveau de saturation. Il est maintenant intéressant de comparer les prédictions de ces modèles avec les mesures d'amplitude d'onde plasma.

\subsection{Mesures DES BANDES LATÉRALES. - Le} moyen privilégié pour mesurer l'amplitude de l'onde plasma est la diffusion Thomson. Ainsi, chacune des deux fréquences qui ont stimulé l'onde plasma, peut se coupler paramétriquement à cette onde pour produire des bandes latérales. Pour l'écart de fréquence qui nous concerne, la bande latérale Stokes se trouve à $11,8 \mu \mathrm{m}$, l'anti-Stokes à $8,7 \mu \mathrm{m}$. L'amplitude des bandes latérales qui sont diffusées vers l'avant, c'est-à-dire dans la même direction de propagation que l'impulsion laser, est une mesure directe de l'amplitude de l'onde plasma.

Malheureusement, l'intensité laser non focalisée est suffisante pour produire aussi un couplage non linéaire à l'extérieur du plasma, probablement dans le milieu laser, de sorte qu'il y a plus de signal à la fréquence des bandes latérales venant du «laser» qu'il n'en est produit par le couplage avec l'onde plasma. Il est alors impossible d'avoir de l'information sur l'onde plasma en mesurant les bandes latérales diffusées vers l'avant.

Par contre il est possible de mesurer les bandes latérales en rétrodiffusion. Ce signal ne peut être une réflection des bandes latérales venant du «laser » car la densité du plasma est $1 \%$ de la densité critique, ni de la rétrodiffusion car l'intensité des bandes latérales est trop faible pour induire un processus stimulé. Les deux mécanismes de couplage qui peuvent générer du signal dans la bande latérale anti-Stokes $(8,7 \mu \mathrm{m})$ sont indiqués à la figure 5 . Ce sont deux mécanismes de couplage résonnant. La figure 5a montre l'onde ionique associée à la rétrodiffusion du $9,56 \mu \mathrm{m}$ se couplant à l'onde plasma « rapide» (dû au couplage des deux ondes lasers parallèles) pour produire une onde plasma rétrodifusée ayant une fréquence $\omega_{\text {pe }}$ et un nombre d'onde $k=2 k_{9}-k_{\text {beat }}=k_{9}+k_{10}$. Le nombre d'onde de ce plasmon peut augmenter par dispersion en propageant vers une région de plus basse densité électronique et se coupler au $9,56 \mu \mathrm{m}$ incident pour produire une onde électromagnétique à $8,7 \mu \mathrm{m}$ en rétrodiffusion. Le mode de couplage illustré figure $5 \mathrm{~b}$ représente le couplage résonnant des deux pompes lasers mais en propagation antiparallèle. L'onde incidente à $10,56 \mu \mathrm{m}$ peut se coupler à l'onde à $9,56 \mu \mathrm{m}$ rétrodiffusée pour produire un plasmon rétrodiffusé à grand nombre d'onde, identique à l'onde produite 
a)

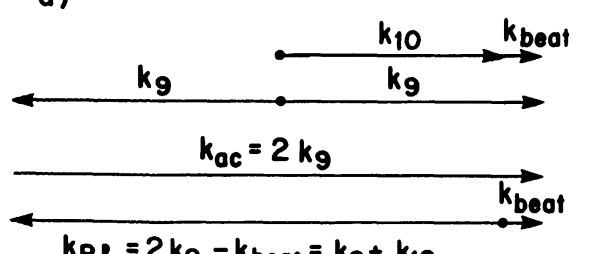

b)

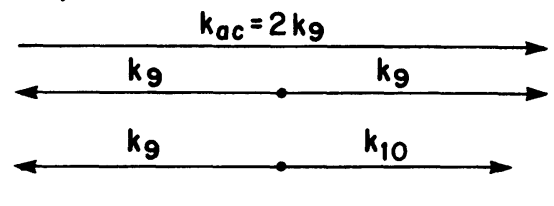

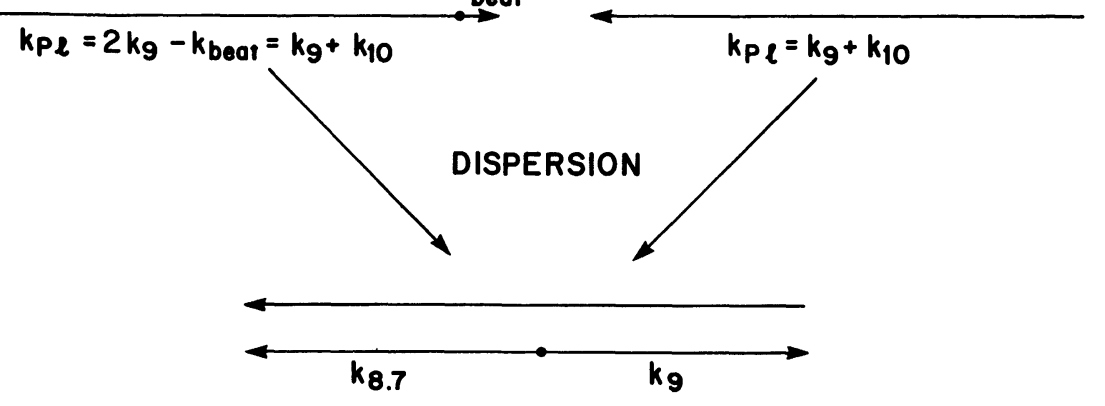

Fig. 5. - Mécanisme de couplage d'ondes pour générer la bande latérale anti-Stokes $(8,7 \mu \mathrm{m})$ en rétrodiffusion.

[Wave coupling mechanism to generate the backscattered anti-Stockes sideband.]

dans le couplage en a. Le couplage de ce plasmon pour produire l'onde à $8,7 \mu \mathrm{m}$ est identique pour les deux schémas de couplage. Il est à noter que la rétrodiffusion Brillouin de l'onde à $9,56 \mu \mathrm{m}$ et le plasmon rétrodiffusé sont communs à ces deux mécanismes de couplage d'ondes. Il n'est pas évident de prédire lequel de ces deux schémas de couplage va dominer la production de bande latérale à $8,7 \mu \mathrm{m}$ en rétrodiffusion.

Ces deux mécanismes de couplage ont été étudiés par Darrow et al. [10] dans le contexte d'études sur la génération de l'onde plasma par battement dans un plasma ayant une modulation spatiale de la densité ionique. Il est à noter que le mécanisme de couplage décrit en 5 a peut aussi se produire quand l'amplitude de l'onde plasma est limitée par la saturation relativiste. La croissance de l'onde plasma n'est pas affectée par la présence de l'onde ionique dans ce régime mais peut s'y coupler pour produire un signal anti-Stokes en rétrodiffusion.

Le paramètre d'importance qui détermine quel mécanisme de saturation limite l'amplitude de l'onde plasma est le niveau de modulation en densité de l'onde ionique. La fraction d'énergie rétrodiffusée par couplage Brillouin stimulé permet une estimation du niveau de modulation ionique. A une intensité laser de $I=4,0 \times 10^{14} \mathrm{Watts} / \mathrm{cm}^{2}$ et une pression de $P=1,4$ torr d'hydrogène $\left(\mathrm{H}_{2}\right)$ produisant une densité électronique de $n_{\mathrm{e}}=1,0 \times 10^{17} \mathrm{~cm}^{-3}$, la fraction d'énergie rétrodiffusée est de $0,4 \%$. Faisant l'hypothèse que la rétrodiffusion par l'onde ionique est cohérente [12], la modulation de densité estimée moyennée sur la longueur du plasma est de $\varepsilon=3,0 \%$. Suivant Darrow et al. [10], l'onde plasma sera dominée par la saturation relativiste si :

$$
a_{1} a_{2} \geqslant \varepsilon^{3}
$$

valide pour $\varepsilon \leqslant 0,1$ où $a_{1} a_{2}=8,1 \times 10^{-3}\left(I_{1} I_{2}\right)^{1 / 2}$ et $I_{\mathrm{i}}$ est en $\left(\times 10^{14} \mathrm{Watts} / \mathrm{cm}^{2}\right)$. Cette relation a été calculée pour une densité électronique de $n_{\mathrm{e}}=$ $1,0 \times 10^{17} \mathrm{~cm}^{-3}$ et une température électronique estimée de $10 \mathrm{eV}$. Pour une intensité laser de $I=4,0 \times 10^{17}$ Watts $/ \mathrm{cm}^{2}$ et une modulation ionique estimée de $3 \%$, la saturation de l'onde plasma est déterminée par l'effet relativiste. Il s'avère donc que le couplage en figure 5a ait une courbe de résonance du type représenté par la figure 4 . Pour ce qui est du couplage en figure $5 \mathrm{~b}$, l'intensité de l'onde rétrodiffusée est suffisamment basse pour que la formule de résonance d'un oscillateur forcé (Eq. (1)) s'applique.

Il est difficile d'estimer l'amplitude de l'onde plasma par des mesures de bandes latérales en rétrodiffusion car c'est un procédé très indirect. Par contre, une mesure de la largeur de résonance pourrait, à priori, identifier lequel des deux mécanismes est en jeu. L'amplitude de l'onde plasma de résonance (petit ou grand nombre d'onde) est proportionnelle à l'amplitude du signal de bande latérale anti-Stokes rétrodiffusée normalisé à l'amplitude de la rétrodiffusion $9,56 \mu \mathrm{m}$.

Les mesures de la bande latérale anti-Stokes $(8,7 \mu \mathrm{m})$ en rétrodiffusion ont été effectuées en utilisant un détecteur $\mathrm{Au}: \mathrm{Ge}$. Un échantillon (4\%) de l'énergie rétrodiffusée est dispersé par un spectromètre à réseau. Un filtre optique, centré à $8,7 \mu \mathrm{m}$, sert à augmenter la réjection. Ces mesures, normalisées par le signal rétrodiffusé à $9,56 \mu \mathrm{m}$ sont présentées sur la figure 4 en fonction de la pression d'hydrogène pour une intensité laser totale de 2,0$4,0 \times 10^{14}$ Watts $/ \mathrm{cm}^{2}$ avec un écart inférieur à $25 \%$ entre l'énergie de chaque longueur d'onde. Cette intensité correspond à une valeur de $a_{1} a_{2}=0,02$. Les barres d'erreur représentent l'écart sur une moyenne de 5 tirs laser à chaque pression. Seuls les 
tirs pour lesquels il y a une bonne synchronisation $(\Delta t \leqslant 0,2 \mathrm{~ns})$ entre les impulsions laser à $9,56 \mu \mathrm{m}$ et $10,56 \mu \mathrm{m}$ sont conservés. Ceci est déterminé à l'aide d'un oscilloscope rapide (Tektronik 7104) et des détecteurs «photon drag » rapides.

Les mesures de bande latérales anti-Stokes ne donnent que l'amplitude relative de l'onde plasma de résonance. Les points expérimentaux ont été superposés à la courbe théorique qui donne le meilleur accord. Il s'agit en l'occurrence de la courbe $a_{1} a_{2}=0,02$ qui correspond à l'intensité laser à laquelle ces mesures ont été faites. L'on remarque tout de suite que les deux spectres sont asymétriques, la courbe expérimentale reproduisant cette asymétrie, bien que par un seul point. La résonance pour le couplage en figure $5 \mathrm{~b}$ est symétrique (Eq. (1)) et nécessite une valeur de $\nu^{*}=\nu / \Delta \omega=0,02$ pour être consistante avec les mesures expérimentales. Cette valeur de la fréquence d'amortissement est plus grande que le taux de croissance de l'onde plasma de sorte que l'onde plasma stimulée dans ce couplage ne peut croître.

Il semble donc que le couplage en figure 5a soit celui qui produit la bande latérale anti-Stokes en rétrodiffusion. Puisque ce couplage implique l'onde plasma rapide, les mesures de $8,7 \mu \mathrm{m}$ en rétrodiffusion permettent une mesure directe de la largeur de résonance de cette onde et, à l'aide du modèle de saturation de Tang et al. [10], un estimé de l'amplitude de saturation. Il est à noter que la largeur de résonance est beaucoup plus grande que la précision sur la pression de remplissage du gaz $\left(\mathrm{H}_{2}\right)$ ionisé par laser qui détermine la densité électronique.

\section{Source d'électrons pour injection.}

Une expérience d'accélération requiert une source d'électrons externe pour l'injection. Pour une onde plasma résonnante à $n_{\mathrm{e}}=1,0 \times 10^{17} \mathrm{~cm}^{-3}$, la vitesse de phase est de $0,995 \mathrm{C}$ ce qui correspond à un électron de 4,7 MeV en énergie. Pour accélérer un électron par une onde plasma de dimension finie $(l=1,5 \mathrm{~mm})$ avec une efficacité maximum, il faut injecter à une énergie moindre, c'est-à-dire autour de 1,0 MeV. Une source d'électrons à haute intensité simplifie la détection des électrons accélérés. Une source pulsée d'une durée du même ordre que la durée de vie de l'onde plasma de résonance ainsi qu'une dimension du faisceau d'électrons voisine de la dimension de l'onde plasma permettent une meilleure discrimination des électrons accélérés.

Un plasma produit par laser $\mathrm{CO}_{2}$ sur cible solide produit une grande quantité d'électrons de haute énergie [13]. La distribution d'énergie est continue et décroissante avec une énergie maximum légèrement supérieure à $1,0 \mathrm{MeV}$ pour une intensité laser de $I=1,0 \times 10^{14}$ Watts $/ \mathrm{cm}^{2}$. L'émission typique est de $10^{8}$ électrons/keV-str à une énergie de $0,5 \mathrm{MeV}$.
La plupart des électrons sont émis dans un cône de $5^{\circ}$ de largeur suivant la normale à la cible durant le temps de montée de l'impulsion laser $(\tau=0,3 \mathrm{~ns})$. La région d'émission se limite à la portion la plus intense de la tache focale d'un diamètre approximatif de $0,1 \mathrm{~mm}$.

Un spectrographe magnétique dipolaire sert à imager les électrons émis du plasma produit sur cible solide jusque dans l'onde plasma de résonance et à les injecter parallèlement à l'onde. Ce spectrographe est représenté sur la figure 6 . La focalisation des électrons dans la direction horizontale et verticale est obtenue par un choix approprié de l'angle des faces d'entrée et de sortie du dipôle magnétique. Ce spectrographe a été conçu pour s'adapter à une source d'électrons produits par laser et a les caractéristiques (calculées) suivantes : l'angle d'acceptance est de $6^{\circ}$, la dimension de la source est de $0,1 \mathrm{~mm}$ par $0,1 \mathrm{~mm}$, la dimension du point image pour des électrons monoénergétiques est de $1,5 \mathrm{~mm}$ par $1,5 \mathrm{~mm}$, l'écart d'énergie au point focal en injectant un large spectre d'énergie est de $\Delta E / E=0,25 \%$, un électroaimant est utilisé permettant la sélection d'électrons dans la gamme d'énergie $0,1 \mathrm{MeV} \leqslant E \leqslant 1,0 \mathrm{MeV}$ au point image et l'espace entre les pôles est de $2,5 \mathrm{~cm}$. La dimension du point image a été vérifiée expérimentalement en utilisant une plaque de phosphore. Le dipôle d'injection et la source plasma produite par impact laser $\mathrm{CO}_{2}$ sur cible solide peuvent ainsi servir de source pulsée d'électrons à une intensité typique de $\simeq 10^{7}$ électrons $/ \mathrm{cm}^{2}-\mathrm{keV}$.

\section{Accélération d'électrons.}

La configuration expérimentale pour effectuer les expériences d'accélération d'électrons est représentée figure 6. Le laser $\mathrm{CO}_{2}$ haute intensité à deux fréquences vient de la droite et est focalisée par un miroir parabolique d'une focale de $60 \mathrm{~cm}$. Ce laser produit le plasma par ionisation de l'hydrogène ( $P=1,4$ torr $)$ puis $\mathrm{y}$ stimule l'onde plasma de résonance. Une seconde impulsion laser $\mathrm{CO}_{2}$ est focalisée sur une cible d'aluminium pour produire un plasma qui sert de source pulsée d'électrons pour l'injection. Le plasma se trouve au point objet du dipôle magnétique. Ces électrons sont dispersés et imagés par le dipôle magnétique jusqu'au plasma produit par le faisceau $\mathrm{CO}_{2}$ principal. Il est à remarquer que la trajectoire de sortie des électrons est parallèle à celle du laser principal. Ceci assure que les électrons sont injectés parallèlement à l'onde plasma de résonance pour être accélérés. Le délai optique entre l'impulsion laser principale et l'impulsion laser secondaire est ajusté pour tenir compte du temps de vol des électrons.

Un spectrographe multicanal est utilisé pour mesurer le nombre d'électrons d'injection et le nombre 


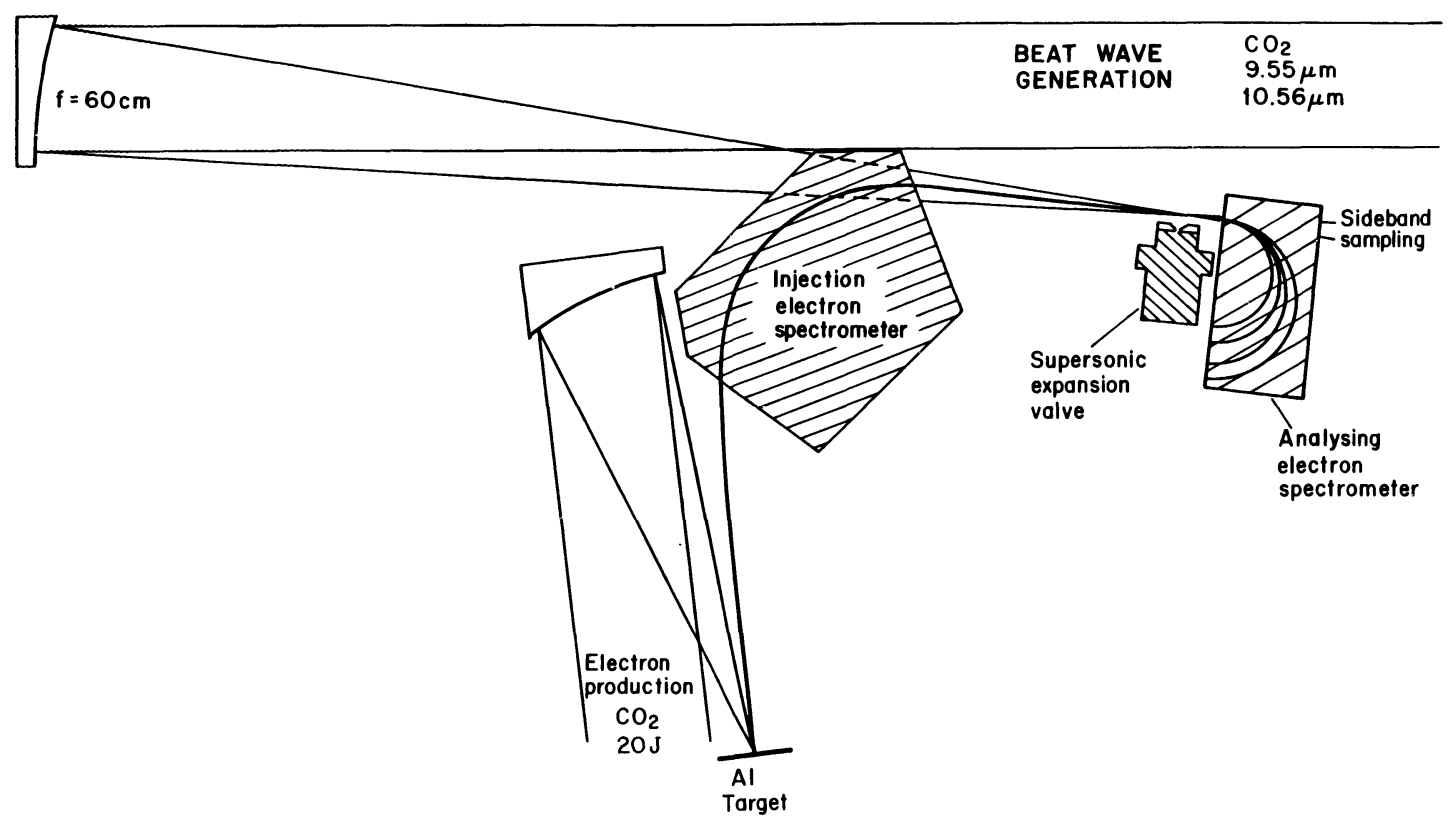

Fig. 6. - Montage expérimental pour l'accélération d'électron par onde de battement.

[Experimental configuration to accelerate electrons by beat-wave.]

d'électrons accélérés. Ce spectrographe possède sept (7) canaux couvrant la gamme d'énergie $0,3 \mathrm{MeV} \leqslant E \leqslant 4,5 \mathrm{MeV}$. Des détecteurs à barrière de surface sont utilisés pour mesurer le nombre d'électrons dans chaque canal. Le signal de ces détecteurs est intégré par un module CAMAC. Le signal de bande latérale anti-Stokes rétrodiffusé est enregistré sur chaque tir. Ce signal sert de mesure relative de l'amplitude de l'onde plasma de résonance permettant d'identifier les tirs où l'onde plasma est susceptible d'accélérer les électrons.

Les expériences d'ionisation laser ont été effectuées en remplissant la chambre à vide à une pression contrôlée. Ce mode de fonctionnement n'est pas acceptable si l'on veut produire, dans la même chambre à vide, des électrons par impact laser sur cible solide ; il y a alors formation d'un plasma devant la cible solide par ionisation du gaz basse pression, diminuant considérablement le nombre d'électrons énergétiques produit. Une solution à ce problème est l'utilisation d'une valve d'expansion supersonique pulsée. Cette valve produit une densité moléculaire locale appropriée pour obtenir une densité électronique de $n_{\mathrm{e}}=1,0 \times 10^{17} \mathrm{~cm}^{-3}$ par ionisation laser tout en maintenant un vide suffisant $\left(P \leqslant 10^{-4}\right.$ torr $)$ dans l'ensemble de la chambre à vide. La valve est pulsée pour $1,0 \mathrm{~ms}$ produisant un faible apport de gaz qui n'est pas suffisant pour augmenter de manière importante la pression de la chambre à vide.

Cette configuration a été utilisée pour des expériences d'accélération d'électrons. Les mesures préliminaires indiquent que des électrons d'injection de $0,6 \mathrm{MeV}$ sont accélérés jusqu'à 2,0 MeV. Puisque la longueur de l'onde plasma de résonance est de $1,5 \mathrm{~mm}$, ceci correspond à une accélération de l'ordre de $1,0 \mathrm{GeV} / \mathrm{m}$.

Il est important de vérifier que les électrons mesurés dans les canaux haute énergie du spectrographe ont bien été accélérés par l'onde plasma de résonance. Nos mesures démontrent qu'il n'y a pas d'électrons de haute énergie pour un laser haute intensité fonctionnant seulement à une longueur d'onde, indiquant que le couplage Raman stimulé vers l'avant n'est pas suffisant pour accélérer des électrons. Pour un laser haute intensité fonctionnant à deux fréquences, aucun électron de haute énergie n'a été détecté lorsque la densité électronique ne correspond pas à la résonance. Il en est de même lorsqu'il n'y a pas d'injection d'électrons indiquant que l'onde plasma n'accélère pas des électrons de basse énergie du plasma supportant l'onde.

\section{Sommaire et conclusion.}

L'ionisation laser de gaz basse pression peut servir de source plasma pour un accélérateur par onde de battement. Bien qu'une intensité laser de l'ordre de $I=1,0 \times 10^{14}$ Watts $/ \mathrm{cm}^{2}$ soit nécessaire, une seule impulsion laser à deux fréquences peut ioniser le gaz et stimuler l'onde plasma de résonance. Pour tester ce concept d'accélération, un plasma produit par impact laser $\mathrm{CO}_{2}$ sur cible solide peut servir de source pulsée d'électrons de haute énergie pour injecter dans l'onde de battement. Des mesures préliminaires d'accélération d'électrons injectés d'une source externe indiquent un gradient d'accélération de $1,0 \mathrm{GeV} / \mathrm{m}$. 


\section{Remerciements.}

Les auteurs veulent souligner l'excellent support technique de M. St-Pierre, F. Levesque,
P. P. Mercier et J. Gauthier. Cette recherche est financée par le Conseil National de la Recherche Scientifique et le Fond FCAR.

\section{Bibliographie}

[1] Laser Acceleration of Particles, ed. C. Joshi, T. Katsouleas, AIP Conference Proceedings No. 130, 1985.

[2] Tajima, T. and Dawson, J. M., Phys. Rev. Lett. 43 (1979) 267.

[3] Clayton, C. E., Joshi, C., Darrow, C., UmstadTER, D., Phys. Rev. Lett. 54 (1985) 2343 and MARTIN, F., JohnSton, T. W., EBrahim, N. E. Phys. Rev. Lett. 55 (1985) 1651.

[4] RAIZER, Yu. P., Laser-Induced Discharge Phenomena (Consultants Bureau, N. Y.) 1977 and HugHes, T. P., Plasmas and Laser Light (John Wiley and Sons, N.Y.) 1975.

[5] Chin, S. L., Yergeau, F., Lavigne, P., J. Phys. B. 18 (1985) L213.

[6] Dangor, A. E., Dymoke-Bradshaw, A. K. L., DySon, A., Garvey, T., Mitchell, I., Cole, A. J., Danson, C. N., Edwards, C. B., EvaNs, R. G., IEEE Trans. Plasma Sci. PS-15 (1987) 161.

[7] Martin, F., Matte, J. P., Pépin, H., Ebrahim, N. A., Proceedings of the 1987 Workshop on new developments in particle acceleration techniques (Orsay, juin) 1987.

[8] Rosenbluth, M. N. and Liu, C. S., Phys. Rev. Lett. 29 (1972) 701 ;

TANG, C. M., Sprangle, P., Sudan, R. N., Phys. Fluids 28 (1985) 1974 ;
Tang, C. M., Sprangle, P., Sudan, R. N., Appl. Phys. Lett. 45 (1985) 375.

[9] Bingham, R., Cairns, R. A., Dangor, A. E., Evans, R. G., MCKinstrie, C. J., MORI, W. B., Plasma Physics and Controlled Fusion 29 (1987) 115 ;

Matte, J. P., Martin, F., Plasma Physics and Controlled Fusion, à paraître, 1987.

[10] Darrow, C., Mori, W. B., Katsouleas, T. Joshi, C., Umstadter, D., Clayton, C. E., IEEE Trans. Plasma Sci. PS-15 (1987) 107.

[11] LANDAU, L., LifChitz, E., Mécanique Classique (Editions de la Paix, Moscou) 1965.

[12] Slusher, R. E., Surko, C. M., Phys. Fluids 23 (1980) 472.

[13] Ebrahim, N. A., Joshi, C., Phys. Fluids 24 (1981) 139 ;

Aithal, S., Lavigne, P., Pépin, H., Johnston, T. W., Estabrook, K., Phys. Fluids, à parấtre.

[14] Matte, J. P., Martin, F., Pépin, H., Ebrahim, N. E., IEEE Trans. Plasma Sci. PS-15 (1987) 173.

[15] Martin, F., Matte, J. P., Pépin, H., Ebrahim, N. E., IEEE Trans. Plasma Sci. PS-15 (1987) 167. 